\title{
Room temperature photonic crystal band-edge lasing from nanopillar array on GaN patterned by nanosphere lithography
}

\author{
Wai Yuen Fu, Kenneth Kin-Yip Wong, and H. W. Choi ${ }^{\text {a) }}$ \\ Department of Electrical and Electronic Engineering, The University of Hong Kong, Pokfulam Road, Hong \\ Kong
}

(Received 7 November 2009; accepted 2 February 2010; published online 18 March 2010)

\begin{abstract}
An ordered GaN nanopillar array fabricated by nanosphere lithography exhibited room temperature photopumped lasing via the photonic crystal band-edge effect. With a monolayer of self-assembled nanospheres as hard mask, the ordered pattern was transferred to the sample to form nanopillars by inductively coupled plasma dry etch. Under pulsed optical excitation, room temperature lasing with a low lasing threshold of $30 \mathrm{~mJ} / \mathrm{cm}^{2}$ was achieved. The dominant lasing peak, centered at 415.6 $\mathrm{nm}$, corresponds to a band-edge mode at the $\Gamma$-point of the band diagram. A $Q$ factor in the range of 600-700, and spontaneous emission coupling factor of 0.021 were evaluated. (C) 2010 American Institute of Physics. [doi:10.1063/1.3353974]
\end{abstract}

\section{INTRODUCTION}

Ordered nanostructures on GaN materials are commonly formed by self-assembled growth, or patterned with a lithographic technique such as electron beam ${ }^{1}$ or nanoimprint. ${ }^{2}$ The incorporation of nanostructure arrays have, depending on the degree of order, been demonstrated to promote light scattering due to surface roughening, or enhance light extraction through the properties of a photonic crystal. ${ }^{3}$ On the other hand, with careful design of the array, an optical cavity can be formed with the aid of the light confinement effects of a photonic crystal, which can be exploited for the realization of surface-emitting laser structures. Recently, a GaN photonic crystal laser patterned by electron beam lithography with blue lasing emission ${ }^{4}$ had been demonstrated. We would like to explore the possibility of achieving the same with a lowcost patterning technique such as nanosphere lithography, a method involving self-assembly of nanosized spherical particles into regular hexagonal closed-packed arrays. Nanosphere lithography offers high throughput processing compared to point-by-point writing with e-beams, and is economical compared to nanoimprinting. In this letter, we report in a self-assembled approach toward fabrication of nanopillar arrays on GaN material, and demonstrate the possibility of achieving low-threshold room temperature lasing via the photonic crystal band-edge effect.

\section{FABRICATION}

The ordered nanopillar structure was fabricated by a two-step process on top of a sample containing InGaN/GaN multiquantum well (MQW) structures. The wafers were grown by metal-organic chemical vapor deposition on a c-plane sapphire substrate. The layer structure is composed of a $25-\mathrm{nm}$-thick GaN buffer layer, a 3- $\mu$ m-thick Si-doped GaN layer, five periods of 2/5-nm-thick InGaN/GaN MQWs, a 50-nm-thick Mg-doped AlGaN layer and topped with a

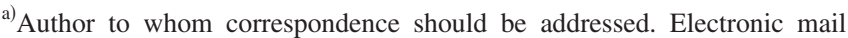
hwchoi@hku.hk. Tel.: (852) 28592693. FAX: (852) 25598738.
}

250-nm-thick Mg-doped GaN layer. A monolayer of $190 \mathrm{~nm}$ silica nanosphere was first coated onto a laser-diced 10 $\times 10 \mathrm{~mm}^{2}$ sample by vertical deposition. ${ }^{5}$ This monolayer of nanosphere, which self-assembled into a hexagonal closepacked array, acts as a hard mask for the subsequent etch step. The sample was then dry-etched by inductively coupled plasma (ICP) using a gas chemistry comprising of 15 SCCM (SCCM denotes cubic centimeter per minute at STP) of $\mathrm{Cl}_{2}$ and $8 \mathrm{SCCM}$ of Ar for $90 \mathrm{~s}$, at coil and plate powers of 350 $\mathrm{W}$ and $135 \mathrm{~W}$, respectively. The pattern was naturally transferred from the hard mask to form ordered hexagonal closepacked nanopillars. The oxide nanosphere residue was then removed by $10 \% \mathrm{HF}$ acid wet etching for $10 \mathrm{~min}$ followed by sonication in acetone for another $10 \mathrm{~min}$. The resultant nanopillar array is illustrated in the field-emission scanning electron microscope (FE-SEM) image in Fig. 1. The height of the nanopillars is around $150 \mathrm{~nm}$, leaving the active MQW region beneath intact.

\section{RESULTS AND DISCUSSIONS}

The optical properties of the nanopillars were evaluated by photoluminescence (PL). A frequency-tripled ND:YLF diode-pumped solid state laser with $349 \mathrm{~nm}$ emission peak and pulse duration of $\sim 4 \mathrm{~ns}$ at $1 \mathrm{kHz}$ repetition rate was used as an excitation source. The nanopillar sample was placed onto a cryogenic stage with temperature control between 77 and $550 \mathrm{~K}$. The emitted signal was collected by a fiber bundle and channeled to a $150 \mathrm{~mm}$ spectrograph (Acton

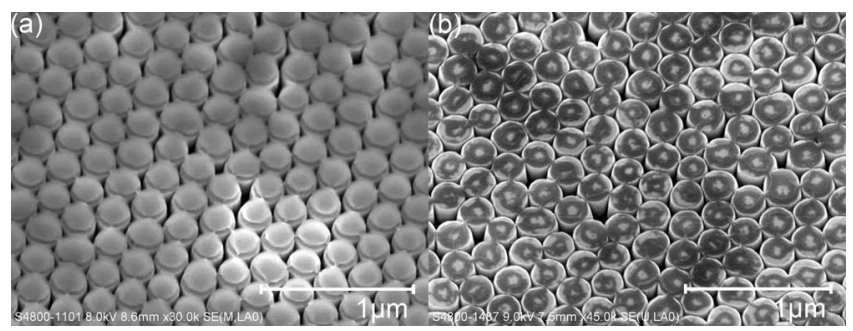

FIG. 1. FE-SEM images showing hexagonal close-packed nanopillars (a) before and (b) after removal of nanosphere layer. 

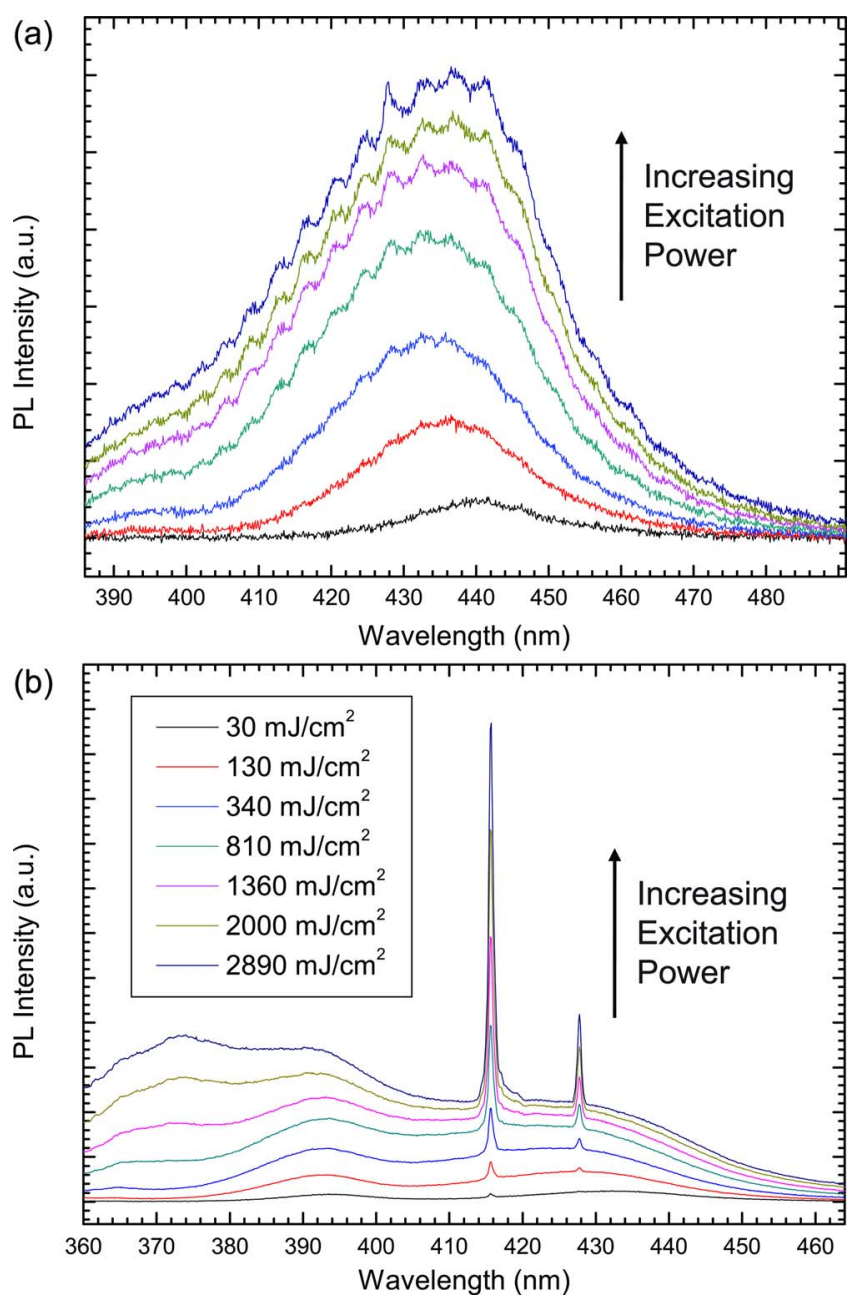

FIG. 2. (Color online) Room temperature PL spectra of (a) as-grown and (b) nanopillar sample under varying excitation energy density.

SP-2150i) and dispersed with a $1200 \mathrm{~L} / \mathrm{mm}$ grating. A Peltier-cooled charge coupled device (CCD) camera (Acton Pixis 256) senses the dispersed light, the entire system has an optical resolution of better than $0.3 \mathrm{~nm}$. The emission image can be simultaneously monitored by a high-resolution CCD camera confocal to the optical path.

The as-grown sample with a PL peak at $\sim 440 \mathrm{~nm}$ exhibits typical MQW spectral characteristics under varying excitation power density as plotted in Fig. 2(a). A blueshift of at most $6.7 \mathrm{~nm}$ when the excitation power density was increased from 30 to $340 \mathrm{~mJ} / \mathrm{cm}^{2}$, followed by a redshift of $4 \mathrm{~nm}$ when the pump power density was further increased to $4.2 \mathrm{~J} / \mathrm{cm}^{2}$. In conjunction with spectral shift, spectral broadening was also observed, with full width at half maximum of the spectral peak increasing by over $12 \mathrm{~nm}$ with increasing excitation powers. Interference fringes across the broadband spectrum were prominent, representing the Fabry-Perot modes due to optical confinement between the GaN/air and GaN/sapphire interfaces.

For the nanopatterned sample, the spectral widths of the primary broadband peak centered at $440 \mathrm{~nm}$ are generally narrower compared to as-grown due to lateral optical confinement effect of nanopillars. Two other additional PL peaks centered at 373 and $393 \mathrm{~nm}$ were detected as shown in Fig.

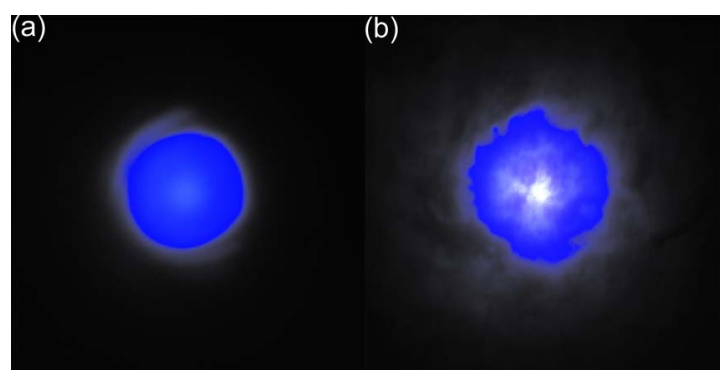

FIG. 3. (Color online) CCD images showing PL emission from (a) as-grown and (b) nanopillar sample under excitation at pump energy density of $340 \mathrm{~mJ} / \mathrm{cm}^{2}$

2(b), representing the PL signal from the exposed $n-G a N$ and p-AlGaN layers deeper in the structure after partial removal of the p-GaN cap layer. The Fabry-Perot oscillations appear to have been suppressed as a result of the textured surface, while spectral broadening upon increasing excitation powers was insignificant compared to the as-grown. Most significantly, two sharp lasing peaks centered at 415.6 and 427.8 $\mathrm{nm}$ are observed. Varying excitation power introduces no obvious shift to the lasing peaks, despite the spectral shift experienced by the broader emission centered at $440 \mathrm{~nm}$ on which the lasing peaks superimposed. At room temperature $(300 \mathrm{~K})$, the quality factor $Q$ of the $415.6 \mathrm{~nm}$ lasing peak is evaluated to be in the range of 600-700.

Figure 3 shows two captured CCD images comparing emission from the as-grown and nanopillar samples under identical excitation energy density of $340 \mathrm{~mJ} / \mathrm{cm}^{2}$. The asgrown sample emits blue light uniformly across the circular region. As for the nanopillar sample, an intense spot of light is observed in the center, surrounded by blue light around it. The spot appears white due to saturation of the detector. Also, the circle is distorted due to the textured surface.

Figure 4(a) shows a plot of integrated PL intensity as a function of excitation power density corresponding to the lasing peak at $415.6 \mathrm{~nm}$. The plot illustrates that the signal intensity grows superlinearly with excitation energy density, with a lasing threshold of $\sim 30 \mathrm{~mJ} / \mathrm{cm}^{2}$. Rearranging the experimental data by putting the PL intensity in logarithmic scale as illustrated in Fig. 4(b), the spontaneous emission coupling factor $\beta$, which is inversely proportional to the difference in PL intensity before and after the lasing threshold on the logarithmic scale, can be estimated to be $2.1 \times 10^{-2}$. This value of $\beta$ can then be used to calculate the Purcell factor $F_{P}$ with the following equation: ${ }^{6}$

$$
F_{P} \simeq \frac{\beta}{1-\beta} .
$$

The Purcell factor $F_{P}$ is determined to be about 2.1 $\times 10^{-2}$, which is then used to derive the effective cavity volume $V_{\text {eff }}$. An equation derived from the Fermi's Golden Rule is given by ${ }^{6,7}$

$$
F_{P}=\frac{3 Q\left(\lambda_{C} / n\right)^{3}}{4 \pi^{2} V_{\mathrm{eff}}},
$$

where $Q$ is the $Q$ factor, $\lambda_{C}$ is the lasing peak wavelength, and $n$ is the refractive index of the material. In our calcula- 

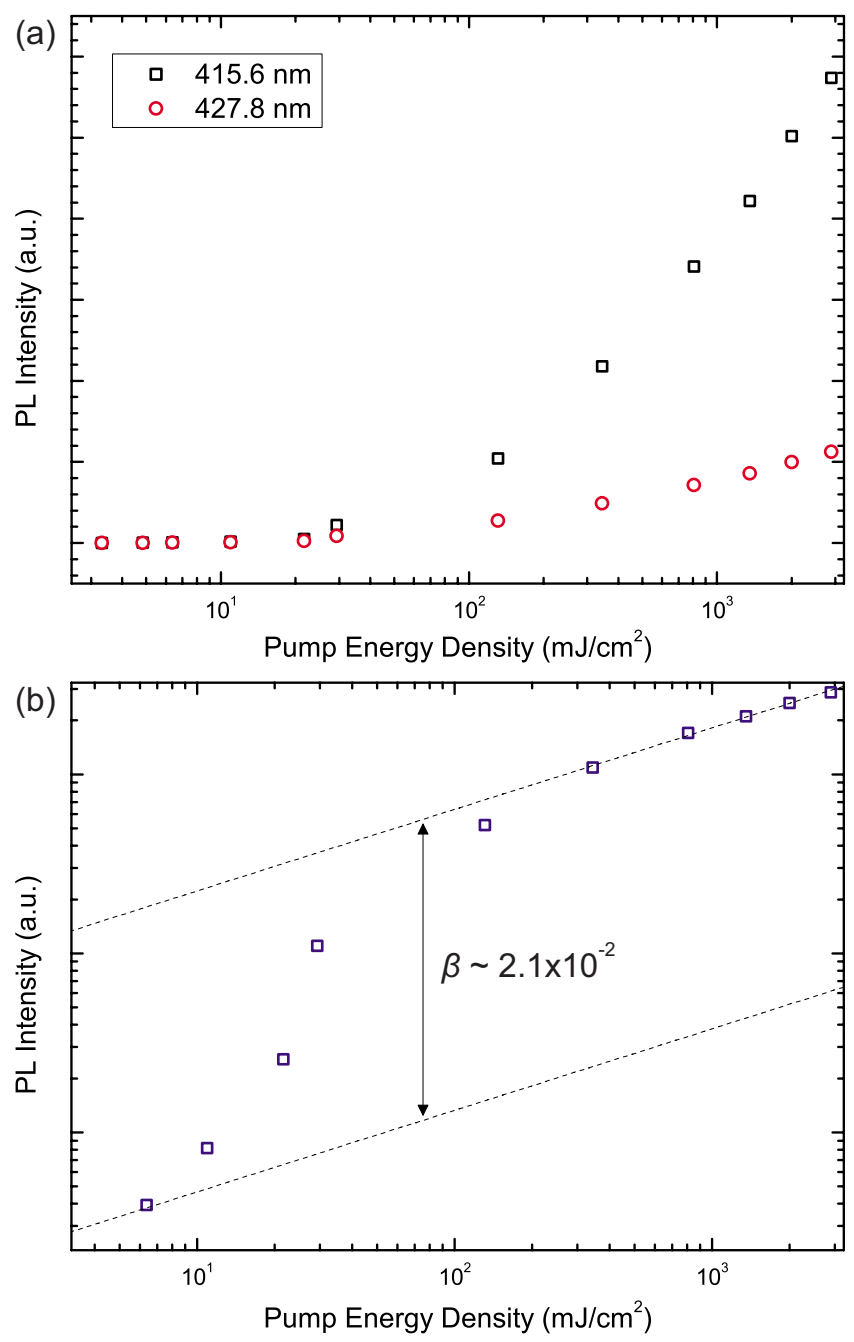

FIG. 4. (Color online) (a) Integrated PL intensity of the two lasing peaks of the nanopillar sample. The open square indicates the $415.6 \mathrm{~nm}$ lasing peak, whereas the open circle indicates the $427.8 \mathrm{~nm}$ lasing peak and (b) integrated PL intensity of the $415.6 \mathrm{~nm}$ lasing peak replotted in logarithmic scale, from which $\beta$ is evaluated.

tion, we use the average value of 646 , for $Q$, lasing peak wavelength $\lambda_{C}$ of $415.6 \mathrm{~nm}$ and refractive index of $\mathrm{GaN}$ is 2.55 . The estimated value of effective cavity volume $V_{\text {eff }}$ is thus $9.8 \mu \mathrm{m}^{3}$. As the Purcell factor is determined to be smaller than unity, the Purcell enhancement effect is thus not present with this photonic crystal structure, which is reasonable since a physical microcavity is absent. Instead, the photonic mode spreads across multiple nanopillars, contributing to the relatively large estimated effective cavity volume. The observed lasing is therefore attributed solely to the photonic crystal band-edge effect.

Our experimentally derived value for the spontaneous emission coupling factor $\beta$, which is of the order of $10^{-2}$, is comparable to recent studies of GaN lasing devices with spontaneous emission coupling factor enhancement, for instance, a vertical cavity surface emitting laser ${ }^{6}$ or a photonic crystal nanocavity light source. ${ }^{8}$ The fabricated nanopillars, unlike other approaches involving deposition, is composed of high-quality single-crystal GaN material, although the dimension of the effective cavity volume may be affected by
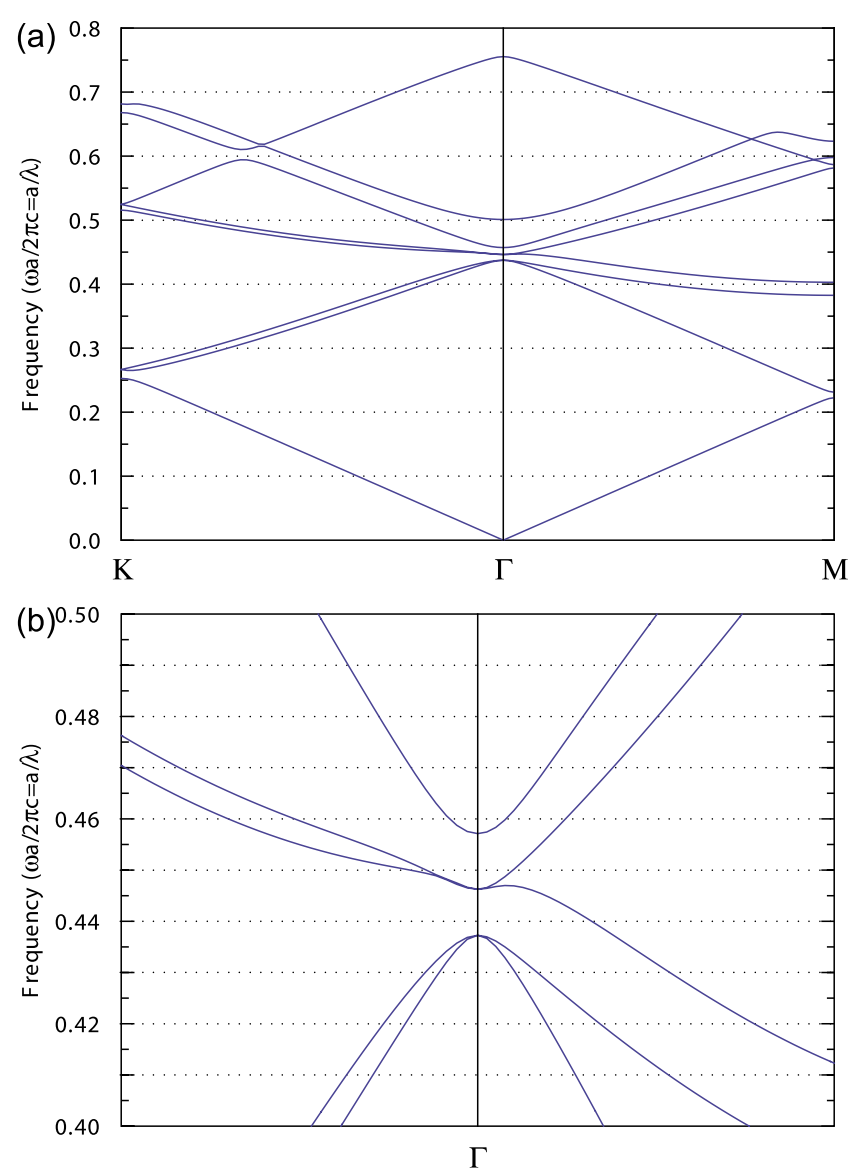

FIG. 5. (Color online) (a) Simulated photonic band diagram of nanopillar array and (b) shows the zoomed-in region around the target band-edges.

the loss induced by surface plasma damage. Also, the presence of MQWs within the nanopillars ensured high gain and thus low-threshold lasing.

To understand the observed lasing phenomenon from the nanostructured sample, we consider the conditions for different possible modes of lasing mechanisms. For Fabry-Perot type lasing, a pair of parallel facet must exist for establishing a resonant standing wave within an optical cavity. However, the fabricated nanopillars are of dimensions smaller than the wavelength of light, resulting in diffraction and enhanced light extraction, which in turn suppresses the occurrence of Fabry-Perot modes. This is verified through the disappearance of interference fringes from the PL spectra of the nanopillar sample. On the other hand, in order to qualify for whispering gallery mode lasing, a circular cavity must exist to provide light confinement for resonance, and that the size of the cavity has to be much larger than the wavelength of light. In our circular nanopillars of subwavelength dimensions, although a whispering gallery mode is supported as predicted from finite-difference time-domain simulations, it is, however, too leaky to acquire sufficient gain. Moreover, since each individual nanopillar is in close contact with neighboring units due to its hexagonal close-packing, any possible whispering gallery mode is likely to be disrupted, as the modes of individual nanopillars will interfere with each other. With other mechanisms ruled out, the photonic crystal 
properties associated with the periodic nanostructure pattern are the most likely causes contributing to the lasing phenomena.

A simulation was carried out using RSoft BANDSOLVE (Ref. 9) to verify the possible lasing positions via the dispersion effect. The plane wave expansion method is used in the simulation, which by solving the vector Helmholtz equation to compute the band structure of the photonic crystal structure modeled with the Bloch's theorem ${ }^{10}$

$$
\hat{\mathbf{L}} \mathbf{u}_{\mathbf{k}}=(i \mathbf{k}+\nabla) \times\left(\frac{1}{\varepsilon(\mathbf{x})}(i \mathbf{k}+\nabla)\right) \times \mathbf{u}_{\mathbf{k}}=\left(\frac{\omega}{c}\right)^{2} \mathbf{u}_{\mathbf{k}},
$$

where the mode function $\mathbf{u}_{\mathbf{k}}=\sum c_{\mathbf{G}, \sigma} \mathbf{e}_{\mathbf{G}, \sigma} \exp (i \mathbf{G} \cdot \mathbf{x})$ is the G, $\sigma$ eigenvector of the Hermitian operator $\hat{\mathbf{L}}, \mathbf{G}$ is the reciprocal lattice vector, $\sigma$ is polarization state, $\mathbf{e}_{\mathbf{G}, \sigma}$ is the unit polarization vector, $c_{\mathbf{G}, \sigma}$ is the corresponding expansion coefficient, $\mathbf{k}=$ wave vector, and $\varepsilon(\mathbf{x})$ is the dielectric function.

Figure 5 displays the simulated photonic band structure. Using a lattice constant of $\mathrm{a}=190 \mathrm{~nm}$, in terms of normalized frequency $(a / \lambda)$ the gain region of the MQWs lies in the range of $0.4-0.465$. With an apparent absence of a photonic bandgap, the lasing phenomenon is expected to be contributed by the photonic band-edge (or dispersion) effect. The three possible lasing points are situated at the band-edge of $\Gamma$-point, with a normalized frequencies of $0.437,0.446$, and 0.457 as shown in Fig. 2(b), where the light wave will be subjected to a maximum feedback due to zero group velocity $(\mathrm{d} \omega / \mathrm{d} k=0)$. Compared with the experimental results, we are not able to observe the lasing peak, which corresponds to normalized frequency of 0.437 . This is caused by blueshifting of the gain region upon high intensity optical pumping.

\section{CONCLUSION}

In summary, we have demonstrated room temperature lasing from GaN nanopillar structures fabricated through nanosphere lithography. The fabrication process made use of self-assembly of nanospheres and a subsequent ICP dry etch. Room temperature lasing under pulsed excitation was achieved, with a lasing threshold of $30 \mathrm{~mJ} / \mathrm{cm}^{2}$, a dominant lasing peak centered at $415.6 \mathrm{~nm}$ and a $Q$ factor in the range of 600-700. The spontaneous emission coupling factor is enhanced to $2.1 \times 10^{-2}$, with an effective cavity volume of $9.8 \mu \mathrm{m}^{3}$. The lasing mechanism was determined to be induced by the photonic band-edge effect of the nanopillar array and this was verified by simulation.

${ }^{1}$ A. David, T. Fujii, R. Sharma, K. McGroddy, S. Nakamura, S. P. DenBaars, E. L. Hu, C. Weisbuch, and H. Benisty, Appl. Phys. Lett. 88, 061124 (2006).

${ }^{2}$ K. J. Byeon, S. Y. Hwang, and H. Lee, Appl. Phys. Lett. 91, 091106 (2007).

${ }^{3}$ W. Y. Fu, K. K. Y. Wong, and H. W. Choi, Appl. Phys. Lett. 95, 133125 (2009).

${ }^{4}$ H. Matsubara, S. Yoshimoto, H. Saito, J. L. Yue, Y. Tanaka, and S. Noda, Science 319, 445 (2008).

${ }^{5}$ R. G. Shimmin, A. J. DiMauro, and P. V. Braun, Langmuir 22, 6507 (2006).

${ }^{6}$ T. C. Lu, C. C. Kao, H. C. Kuo, G. S. Huang, and S. C. Wang, Appl. Phys. Lett. 92, 141102 (2008).

${ }^{7}$ J. M. Gerard and B. Gayral, J. Lightwave Technol. 17, 2089 (1999).

${ }^{8}$ C. F. Lai, P. Yu, T. C. Wang, H. C. Kuo, T. C. Lu, S. C. Wang, and C. K. Lee, Appl. Phys. Lett. 91, 041101 (2007).

${ }^{9}$ BandSOLVE (RSoft Design Group).

${ }^{10}$ J. D. Joannopoulos, S. G. Johnson, J. N. Winn, and R. D. Meade, Photonic Crystals: Molding the Flow of Light, 2nd ed. (Princeton University Press, Princeton, 2008) 